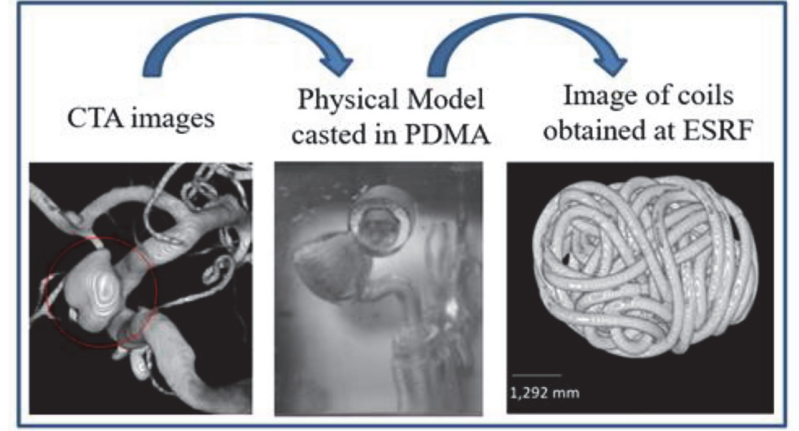

Abstract P-056 Figure 2 Patient A's CFD model. Table shows different approaches to porosity. 'Coils resolved' is the

simplification introduces error into CFD simulations that may affect accuracy. We developed an improved method of representing the coil mass in CFD simulations to reduce such error. This method characterizes the porous spatial distribution of the coil mass using 3D-printed PDMA models of seven patient-specific aneurysms treated with commercially-available coils and scanned using synchrotron microtomography. This provides high-resolution, 3D images of the coil mass geometry (figure 1). Images were segmented to analyze the porosity distribution along the aneurysm radius. Permeability and inertial factor parameters were used to construct a mathematical representation of the coil mass that did not require microtomographic scanning. This was then incorporated into CFD simulations and compared to simulations of the same aneurysms which incorporated the actual microtomographic coil geometry (figure 2). The results show that porous distribution (varying between 0.6 and 0.95 ) leads to improved permeability gradients, which reduced the error in the mean velocity within the aneurysm dome to lower than 15\%, substantially better than that seen with traditional homogenous porous media approaches. Our method thus better represents the effect of the coil mass on intraaneurysmal hemodynamics as compared to the standard approach used in many CFD simulations. Our method could be applied to future CFD studies of coiled aneurysms, without microtomographic scanning, to improve the accuracy of hemodynamic calculations and predict treatment outcome.

Disclosures J. Romero Bhathal: None. F. Chassagne: None. L. Marsh: None. M. Levitt: 1; C; Medtronic, Stryker. 2; C; Medtronic, Metis Innovative. 4; C; eLoupes, Cerebrotech, Synchron. D. Bass: None. J. Keen: None. C. Geindreau: None. A. Aliseda: None.

\section{P-057 INITIAL EXPERIENCES USING THE WAHOO DUAL MODE GUIDE CATHETER}

J Vargas*, M Chaudry, A Turk, R Turner. Neurosurgery, Prisma Health - Upstate, Greenville, SC

\subsection{6/neurintsurg-2021-SNIS.93}

Introduction The Q'Apel Medical Wahoo guide catheter is a 7 French guide catheter approved by the FDA in September of 2018 with an 0.072 ' inner diameter and the ability to alternate between tracking and support modes. Tracking mode allows the catheter to navigate distally, and once in support mode, catheter positioning remains extremely stable, mitigating the need for a tri-axial 8 French system. We report our experiences with the Wahoo guide catheter in a variety of embolization procedures.

Methods A retrospective chart review was performed of cases in which the Wahoo catheter was used as a guide from February 2020 to January 2021. Patient demographics, lesions treated, side of lesion, treatment technique, access site, vessel characteristics, devices used, and peri and post procedural complications were collected.

Results 46 consecutive cases were identified. 32 patients were female (69\%), and average age was 55 ( \pm 13 , range 17-83) years. 41 cases were aneurysm embolizations (89.1\%), 3 were MMA embolizations for cSDH (6.5\%), and there was one case of an AVM rupture (2.2\%) and one case of balloon angioplasty for ICAD (2.2\%). 20 cases were treated from the left $(43.5 \%), 24$ were treated from the right $(52.2 \%)$, and in 2 MMA embolizations both sides were treated (4.3\%). 33 cases were performed with femoral access (71.7\%), 12 via radial access $(26.1 \%)$. In 14 cases, there was vessel tortuosity (30\%): 4 severe (8.7\%), 3 moderate (6.5\%), and 7 mild $(15 \%)$. Of the 39 cases in which the guide was placed in the ICA $(84.8 \%), 33$ were placed in the petrous ICA or distal to it $(84.6 \%)$.

There were no instances of guide prolapse from the segment in which it was placed. A variety of treatment modalities were employed. 15 coil (33.6\%), 2 coil plus flow diversion (4.3\%), 2 coil plus stenting (4.3\%), 19 flow diversion (41.3\%), 2 WEB (4.3\%), 4 Onyx embolizations (8.7\%), and 1 balloon angioplasty $(2.2 \%)$ were performed.

There were two procedural complications: one dissection due to wire navigation and unrelated to the Wahoo guide catheter (2.2\%), and one dissection which in which the causal relationship was undetermined (2.2\%). There were no longterm neurological sequelae in either case. There were no access site complications. All procedures were successfully completed with no need to change to another guide.

Conclusion The Wahoo catheter is an effective guide catheter than can accommodate a diversity of devices, can be safely navigated into distal vasculature, and be used in a variety of embolizations in lieu of an 8 French based tri-axial system.

Disclosures J. Vargas: 2; C; Corindus, Cerenovus, Integra, Medtronic. 4; C; Truvic, Ceretronic, Visom. M. Chaudry: 2; C; Q’Apel. 4; C; Q’Apel. A. Turk: 2; C; Q’APel, Balt, Cardinal Consulting, Cerebrotech, Medtronic, Endostream, Microvention, Siemens, Three Rivers Medical, Vastrax, Shape Memory, Stryker, Serenity, 880 Medical, Pipetherapeautics. 4; C; Imperitive Care, Cerebrotech, Endostream, Shape Memory, Synchron, Serenity, Echovate, RIST, APAMA. R. Turner: 2; C; Q'Apel, Cerenovus, Medtronic, Balt, Endostream. 4; C; Q’APel. 5; C; Seimens. 6; C; MUSC.

\section{P-058 TARGET ULTRASOFT AND NANO COILS FOR THE TREATMENT OF SMALL BRAIN ANEURYSMS}

${ }^{1}{ }^{G}$. Jindal ${ }^{*}$, ${ }^{1}$ R. Almardawi, ${ }^{1}$ D. Gandhi, ${ }^{1}$ T. Miller, ${ }^{2}$ S. Satti, ${ }^{3}$ F. Hui. ${ }^{1}$ University of Maryland Medical Center, Baltimore, MD; ${ }^{2}$ Christiana Care Medical Center, Newark, DE; ${ }^{3} J o h n s$ Hopkins Hospital, Baltimore, MD

\subsection{6/neurintsurg-2021-SNIS.94}

Purpose The ULTRA Registry is an multicenter, national, prospective study designed to assess aneurysm occlusion rates and safety profile of the Target ${ }^{\circledR}$ Ultrasoft and Nano coils (Stryker 